\title{
Free-electron-like bulk and surface states for $\mathrm{Zn}(0001)$
}

\author{
F. J. Himpsel and D. E. Eastman \\ IBM Thomas J. Watson Research Center, Yorktown Heights, New York 10598 \\ E. E. Koch \\ Deutsches Elektronen-Synchrotron, Notkestrasse 85, 2000 Hamburg 52, West Germany
}

(Received 22 December 1980)

\begin{abstract}
Energy versus momentum $\left(k_{\|}, k_{1}\right)$ energy-band dispersions for $s, p$-like bulk and surface states of a $\mathrm{Zn}(0001)$ surface have been determined using angle-resolved photoemission with synchrotron radiation. We find nearly-free-electron (NFE) bulk $s, p$ bands with an inner potential of $-15.4<V_{0}<-13.5 \mathrm{eV}$ (below the vacuum level). At the zone boundary in the [0001] direction, the occupied $s, p$ bands have a $p$-like critical point $\left(\Gamma_{3}^{+}\right)$at $-3.9 \mathrm{eV}$ below $E_{F}$ and an $s$-like critical point $\left(\Gamma_{4}^{-}\right)$at $-2.9 \mathrm{eV}$, while the NFE final states are found at $+46 \mathrm{eV}$. These NFE final states appear to have slightly different energies $(\sim 2 \mathrm{eV})$ for transitions from occupied $s$ and $p$ states which can be described by an effective $s$ - and $p$-dependent inner potential or by multiple band effects. A fully symmetric $\left(\Lambda_{1}\right) s, p_{z}$-like surface state is found at $-3.6 \mathrm{eV}$ in the $\Gamma_{3}^{+}-\Gamma_{4}^{-}$band gap; the band dispersion $E$ vs $\overrightarrow{\mathrm{k}}_{\|}$of this state has been measured over its full range of existence. The final-state lifetime broadening $(2 \Gamma \sim 4.5 \mathrm{eV}$ at $+37 \mathrm{eV}$ and $2 \Gamma \sim 7 \mathrm{eV}$ at $+54 \mathrm{eV}$ final-state energies, respectively) is reflected in large widths $(\sim 2-3 \mathrm{eV})$ of bulk $s, p$-band emission peaks. Surface-state peaks are much narrower $(0.7 \mathrm{eV})$ and are limited only by initial-state lifetime broadening.
\end{abstract}

\section{INTRODUCTION}

Zinc is a suitable material to study $s, p$-like states in metals. The $d$-like states ${ }^{1,2}$ lie at $\sim 10$ $\mathrm{eV}$ below the Fermi level $\left(E_{F}\right)$ and leave a wide energy range to study $s, p$-like valence states. In particular, the relation between the free-electronlike bulk $s, p$ bands and $s, p$-like surface states can be seen. Such states have been found in $s, p$ gaps for many noble metals, transition metals, and simple metals (see Ref. 3).

We have studied the dispersions of $s, p$-like bulk bands in $\mathrm{Zn}$ from $\sim 8 \mathrm{eV}$ below $E_{F}$ to $70 \mathrm{eV}$ above $E_{F}$ using standard band-mapping methods (see Ref. 4). We find that $s$-like states are higher in energy relative to $p$-like states by $\sim 1 \mathrm{eV}$ near the zone boundary. Also, we have been able to determine the dispersion of the $\Lambda_{1}$-symmetry surface state which is located in the $s, p_{z}$ band gap over its full range of existence. The influence of final-state lifetime broadening on the photoelectron spectra of $s, p$ states is found to be large for bulk states but much less so for surface states.

\section{EXPERIMENTAL}

For our measurements, a two-dimensional display-type electron spectrometer was used. ${ }^{5}$ Synchrotron radiation from the $240-\mathrm{MeV}$ storage ring Tantalus $I$ at the Univer sity of Wisconsin in Madison monochromatized by a $1.5-\mathrm{m}$ toroidal grating monochromator served as a tunable light source. Spectra were taken from a $\mathrm{Zn}(0001)$ surface with mixed $s, p$-light polarization. The surface was prepared by sputter etching and annealing, and was characterized by low-energy electron diffraction (LEED) and Auger electron spectroscopy. For $\mathrm{Zn}(0001)$, a work function of $e \Phi=4.4 \mathrm{eV}$ was determined. All energies are referenced to the Fermi level $E_{F}$ except for the inner potential $V_{0}$, which is measured with respect to the vacuum level following the conventional notation.

\section{BAND DISPERSIONS AND LIFETIMES}

A. Experimental results

Figure 1 gives an overview of photoelectron spectra for normal emission from a $\mathrm{Zn}(0001)$ surface, measured at different photon energies $h \nu$. Three-dimensional bulk bands exhibit an energy dispersion with $h \nu$ (see tic marks) because the momentum perpendicular to the surface, $k_{\perp}$, changes with $h \nu$ for direct interband transitions. ${ }^{4}$ The lower part of the $s, p$ band exhibits a maximum at an initial energy of $-3.9 \mathrm{eV}$ for $h \nu=50.4$ $\pm 1 \mathrm{eV}$. This corresponds to the critical point $\Gamma_{3}^{+}$ which has $p_{z}$ symmetry (cf. Fig. 2). At this point, the bulk $s, p$ band cannot be seen as a separate structure, but it is clear that it comprises a large fraction of the background under the sharp surfacestate peak at $-3.6 \mathrm{eV}$ (to be discussed).

As the photon energy decreases from $h \nu=45 \mathrm{eV}$, the bulk $s, p$-band transition above the surface state is seen in Fig. 1 to disperse towards $E_{F}$. There is a Fermi-level crossing at $h \nu=37.2 \pm 0.4$ eV. A second Fermi-level crossing of this $s, p$ band can be determined to occur at $h \nu=54 \pm 1.5$ $\mathrm{eV}$, where the intensity at $E_{F}$ has a relative maximum (see tic mark).

The two-dimensional surface state remains stationary at $-3.6 \mathrm{eV}$ for all $h \nu$ in Fig. 1 since it is spatially localized at the surface and has no well-defined $k_{\perp}$. However, matrix elements for 


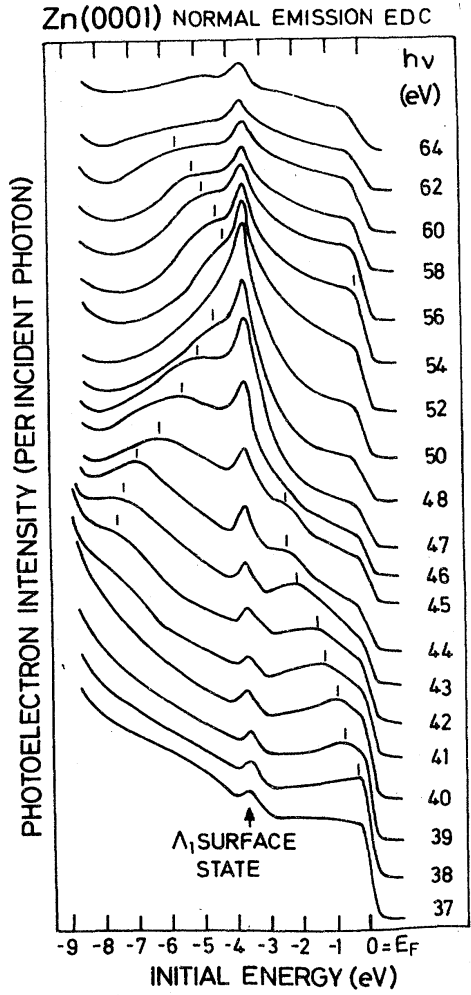

FIG. 1. Photoelectron spectra in normal emission from $\mathrm{Zn}(0001)$ for various photon energies $h \nu$. Interband transitions from the bulk $s, p$ band are shown with tic marks. The $s, p_{z}$-like $\Lambda_{1}$ surface state is seen at -3.6 eV.

excitation into bulklike final states depend on the $k_{\perp}$ of the final state; maximum transition intensities are found to occur for final states near $F_{4}^{*}$ $(+46.5 \mathrm{eV})$ which are located in $\overrightarrow{\mathrm{k}}$ space near the bulk critical points at the top and bottom of the $s, p$-band gap (i.e., near the points $\Gamma_{3}^{+}=-3.9 \mathrm{eV}$, $\left.\Gamma_{4}^{-}=-2.9 \mathrm{eV}\right)$. This has been pointed out by Louie et al. (Ref. 6) for the analogous surface state on $\mathrm{Cu}(111)$.

\section{B. Energy bands along $\Gamma \Delta A$}

From the data in Fig. 1, we have derived the energy-band dispersions along the line $\Gamma \Delta A$ in $\overrightarrow{\mathrm{k}}$ space shown in Fig. 2 (left-hand side). Open circles and open squares are for bulk states, while full circles in Fig. 2 are for the $\Lambda_{1}$ surface state which does not disperse along $\Gamma \Delta A$. The momentum has been obtained from the measured finalstate energy using semiempirical final bands (for a review of the method see Ref. 4). Above a final energy of $30 \mathrm{eV}$, the bands have been taken to be free-electron-like parabolas, but with different energy offsets for transitions from initial $s$ - and

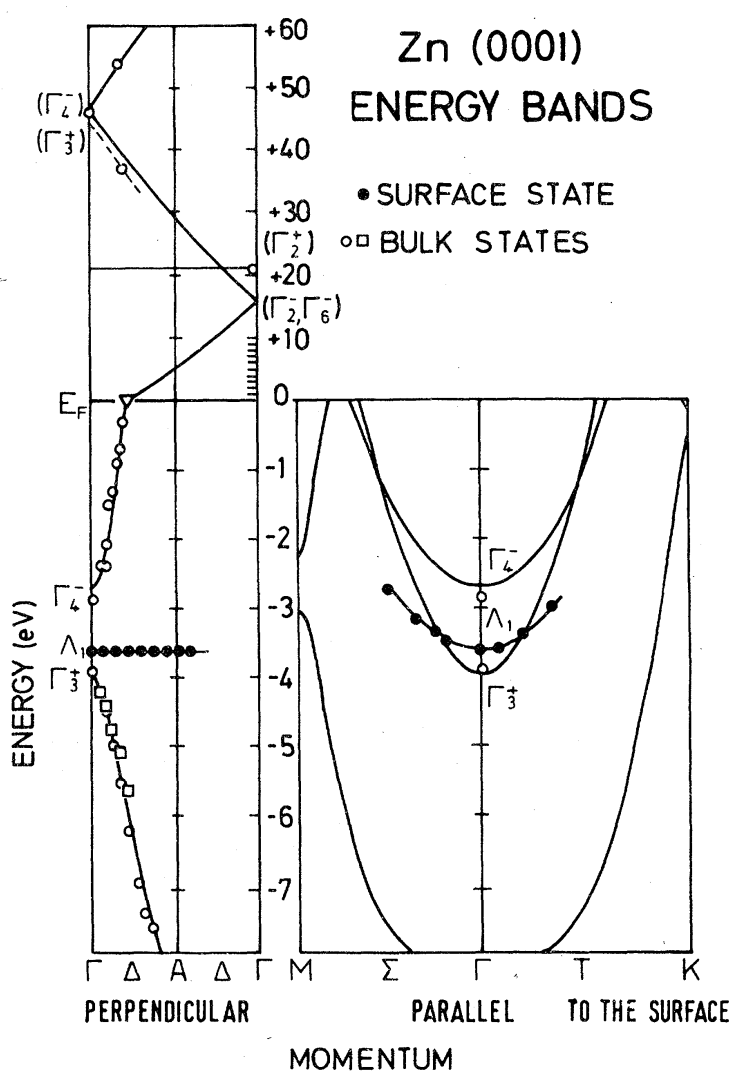

FIG. 2. Summary of energy-band dispersion data for the $s, p$ states in $\mathrm{Zn}$ (for $d$ states; see Ref. 1 ). The full lines below $E_{F}$ are from the calculation of Juras $e t a l$. (Ref. 2). The left-hand side of this figure comprises the normal emission data which cover the [0001] axis $\left(\Gamma A \Gamma=1.27 \AA^{-1}\right)$. The bands have been unfolded such that they are analogous to the bands along $\Gamma L$ in an fcc lattice (see Ref. 4), e.g., the points $\Gamma_{3}^{+}\left(\Gamma_{4}^{-}\right)$correspond to the $L_{2}^{\prime}\left(L_{1}\right)$ points of an fcc lattice. The right-hand side shows the surface-state dispersion perpendicular to the [0001] axis and the corresponding bulk band gap which is spanned by the bands along $\Gamma K$ and $\Gamma M$, respectively $\left(\Gamma K=1.57 \AA^{-1}, \Gamma M=1.36 \AA^{-1}\right)$.

$p$-like states. The bottoms of these parabolic bands (with inner potentials $V_{0}$ ) have been found experimentally by using the Fermi-level crossings $\left(k_{F}=0.42 \Gamma A\right.$, see Ref. 7$)$ at $h \nu=37.2$ and 54 $\mathrm{eV}$, and the extremum in the band dispersion at $h \nu=50.4 \mathrm{eV}$ (initial energy $=-3.9 \mathrm{eV}$ ).

We find that the lower occupied branch of the $s, p$ band (joining the $p_{z}$-like point $\Gamma_{3}^{+}$at $-3.9 \mathrm{eV}$ ) is excited into a band with $V_{0} \simeq-13.5 \mathrm{eV}$ (full lines in Fig. 2). Because of dipole-selection rules, we attribute $s$ character $\left(\Gamma_{4}^{-}\right.$symmetry at $\left.+46.5 \mathrm{eV}\right)$ to this final band. An additional final band (identified from the Fermi-level crossing at $h \nu=37.2$ $\mathrm{eV}$, dashed in Fig. 2) can be described by a nearly-free-electron ( $\mathrm{NFE}$ ) final band with $p_{z}$-like 
character which has a deeper inner potential $\left(V_{0}\right.$ $=-15.4 \mathrm{eV})$. Multiple final-state bands are not uncommon. For example, free-electron-like final bands which run almost parallel like the dashed and full lines in Fig. 2 have been calculated by a LEED (low-energy electron diffraction)-type calculation for $\mathrm{Cu}(111)$ (see Ref. 8). The $\Gamma$ points near $+45 \mathrm{eV}$ from $\mathrm{Zn}(0001)$ correspond to the $L$ points near $+70 \mathrm{eV}$ for $\mathrm{Cu}(111)$ when account is taken of differences in the lattice constants and the inner potentials. In the $\mathrm{Cu}(111)$ calculation (Ref. 8), the $L$ points are split by $\sim 2 \mathrm{eV}$ as well. We suggest that the splitting of the final bands of $\sim 2 \mathrm{eV}$ in $\mathrm{Zn}$ is due, in part, to their different symmetry character.

Below $+30 \mathrm{eV}$ the final bands are less free-electron-like along the threefold axis, as has been demonstrated for $\operatorname{Pd}(111)$ (see Ref. 9). Therefore, we present only a tentative band topology which is derived from previous experience with transition metals (see Refs. 4 and 9) and from data involving the $\mathrm{Zn} 3 d$ bands which are published elsewhere (Ref. 1). Basically, there exists a $p$-like $\Gamma$ point $\left(\Gamma_{2}^{-}, \Gamma_{6}^{-}\right)$at $\sim 16 \mathrm{eV}$ and an $f$-like $\Gamma$ point $\left(\Gamma_{2}^{+}\right)$at $21 \mathrm{eV}$. The latter can be identified from a resonance in the intensity of the lower $d$ band (see Ref. 1).

The $\Gamma_{3}^{+}-\Gamma_{4}^{-}$band gap in which the surface state exists can be seen more directly in Fig. 3 at a photon energy $h \nu=26 \mathrm{eV}$, for which the surface state has a relative minimum in its cross section (see Refs. 6 and 10). When the residual surfacestate emission is subtracted (dashed curve), one clearly sees a dip in the energy distribution with turning points at $\mathbf{- 2 . 9}$ and $-3.9 \mathrm{eV}$. This dip is attributed to the low density of initial states in the $\Gamma_{3}^{+}-\Gamma_{4}^{-}$band gap. In this spectrum indirect transitions predominate since direct interband bulk transitions cannot occur (see also the spectrum at $h \nu=37 \mathrm{eV}$ in Fig. 1). Only evanescent final

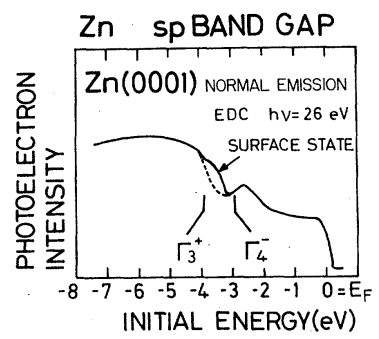

FIG. 3. Photoelectron spectrum for normal emission from $\mathrm{Zn}(0001)$ at $h \nu=26 \mathrm{eV}$, where the $\Lambda_{1}$ surface state has a minimum in its cross section. The dashed line shows the spectrum after the residual surface-state contribution has been subtracted. The $\Gamma_{3}^{+}-\Gamma_{4}^{-}$band gap can be seen as a dip in the photoelectron spectrum from the bulk (dashed curve). states contribute which do not require conservation of the momentum $k_{\perp}$, plus possible surface roughness effects, etc., which are not strongly energy or momentum dependent. This is reflected by the fact that the spectra do not change when $h \nu$ is varied by $\sim \pm 10 \mathrm{eV}$ around $h \nu=26 \mathrm{eV}$. For $\Gamma_{4}^{-}$, our experimental value of $-2.9 \mathrm{eV}$ compares to a calculated value of $-2.7 \mathrm{eV}$ (Refs. 2 and 7), for $\Gamma_{3}^{+}$the experimental value is $-3.9 \mathrm{eV}$ and calculated values are $-4.0 \mathrm{eV}$ (Ref. 2) and $-4.5 \mathrm{eV}$ (Ref. 7).

\section{Surface-state dispersion}

As shown in Fig. 4 for off-normal photoemission data, the surface state disperses upwards when the momentum parallel to the surface $\vec{k}_{\|}$is increased. A summary of this surface state $E$ vs $\vec{k}_{\|}$ dispersion is given on the right-hand side of Fig. 2. The underlying bulk band gap $\left(\Gamma_{3}^{+}-\Gamma_{4}^{-}\right.$at $\left.\vec{k}_{\|}=0\right)$ shrinks with increasing $\overrightarrow{\mathrm{k}}_{\|}$while its center of gravity moves upwards. This can be seen from the calculated bands along $\Gamma M$ and $\Gamma K$, respectively (Ref. 2; see solid lines in Fig. 2) which delineate the gap in the projected band structure. The surface state disperses less than the center of gravity of the band gap and extends into the region where bulk bands exist. At this point, the surface state turns into a surface resonance which gets damped quickly (see Fig. $4, \theta=10^{\circ}$ ) with increasing overlap with bulk states. A very similar behavior has been calculated self-consistently for the analogous surface state on $\operatorname{Pd}(111)$ (Ref. 11). For $\mathrm{Pd}(111)$ it has not been possible to observe the surface state with photoemission since it is

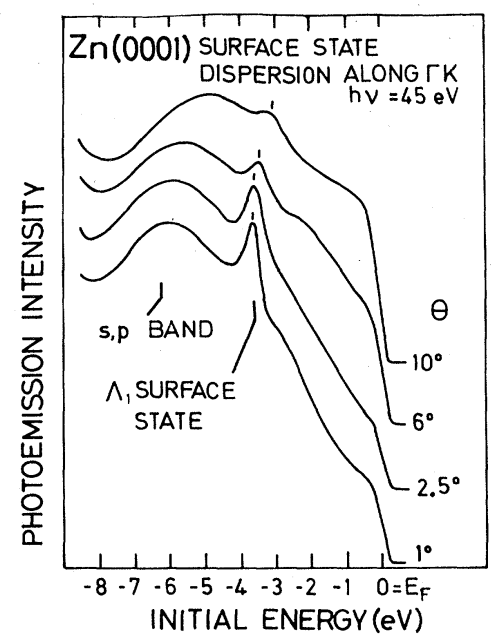

FIG. 4. Photoelectron spectra for various escape angles $\theta$ (from the sample normal) at a fixed photon energy $h \nu=45 \mathrm{eV}$. The dispersion of the $s, p$-like surface state with $\vec{k}_{\|}$can be seen (see tic marks). At $\theta=6^{\circ}$ the surface state crosses the boundary of the bulk band gap. 
unoccupied. Also, in noble metals such as $\mathrm{Cu}(111)$ (Refs. 10 and 12), the surface state disperses above the Fermi level (and is not observed) before it becomes damped by coupling to bulk states.

\section{Lifetimes}

The large width of the interband transitions from the bulk $s, p$ band [ $2 \mathrm{eV}$ full width half maximum (FWHM), see Fig. 1] contrasts to the much sharper surface state $(\sim 0.7 \mathrm{eV}$ FWHM). This is due to final-state lifetime broadening (due to electron-hole pair production by the photoelectron) which smears transitions over a certain range of $k_{\perp}$ values (see Ref. 10). The bulk $s, p$ band has a large slope $\partial E / \partial k_{\perp}$ and is thus strongly affected by final-state lifetime broadening, whereas the surface state has no dispersion with $k_{\perp}$ and exhibits mainly initial-state lifetime broadening (Auger lifetime of the hole). We can measure directly the final-state lifetime broadening by determining the width of transitions from initial states at $E_{F}$ into the final bands (see Ref. 13). For the Fermi-level transitions at $h \nu=37.2 \mathrm{eV}$ $(54 \mathrm{eV})$, we find widths FWHM of $4.5 \mathrm{eV}(7 \mathrm{eV})$, respectively. The final-state lifetime broadening is demagnified by the ratio of slopes for initial and final bands, which is about $\frac{1}{3}$ (see Ref. 10; the contribution due to the initial-state lifetime of $\leqslant 0.7 \mathrm{eV}$ is much smaller). Thus, we are able to explain the large width of the $s, p$ interband transitions as being mostly due to final-state lifetime broadening.

There are some suggestions based on recent data for $\mathrm{Cu}(111)$ (Ref. 14) which seem to give incorrect bulk band dispersions and an intensity transfer from the $s, p$ band to the $\Lambda_{1}$ surface state for transitions to final bands near the $L$ point at $\sim+70 \mathrm{eV}$. In $\mathrm{Cu}$, only a $2-\mathrm{eV}$-wide window is accessible for transitions from the $s, p$ band (between the $d$ bands and $E_{F}$ ) which is comparable to the width of the $s, p$-band transitions at the photon energies under consideration. In $\mathrm{Zn}$, we have a much larger window ( $9 \mathrm{eV}$ wide) and find no anomalies. In particular, the $s, p$-band dispersion mapped from final states below the $\Gamma_{4}^{-}$ point at $+46.5 \mathrm{eV}$ (open circles in Fig. 2) matches exactly the dispersion determined from final states above this $\Gamma$ point (open squares in Fig. 2) and agrees with the calculated bulk band. For $\mathrm{Cu}(111)$ the $s, p$-band dispersion mapped from final states at about $+80 \mathrm{eV}$ in Ref. 14 disagreed with the band dispersion determined at low photon energies and with calculations. For $\mathrm{Zn}(0001)$ we see no intensity transfer from the bulk $s, p$ band to the surface state near $\Gamma_{4}^{-}=+46.5 \mathrm{eV}$. Rather, the bulk $s, p$ band is seen as a fairly smooth background emission because of its larger width. For $\mathrm{Cu}(111)$ the bulk $s, p$-band emission is much harder to estimate because of the narrow observation window of about $2 \mathrm{eV}$. A different assumption for the line shape of the bulk emission may resolve the discrepancies between the bulk band dispersions determined at high and low photon energies, respectively.

\section{ACKNOWLEDGMENTS}

We would like to acknowledge the help of J. J. Donelon, Al Marx, and the support of the U. W. Synchrotron Radiation Center. This work was supported in part by the Air Force Office of Scientific Research (AFOSR), United State Air Force, under Contract No. F49620-80-C0025.
${ }^{1}$ F. J. Himpsel, D. E. Eastman, E. E. Koch, and A. R. Williams, Phys. Rev. B 22, 4604 (1980).

${ }^{2}$ G. E. Juras, B. Segall, and C. B. Sommers, Solid State Commun. 10, 427 (1972).

${ }^{3}$ See, e.g., P. O. Gartland and B. J. Slagsvold, Phys. Rev. B 12, 4047 (1975); P. Heimann, H. Neddermeyer, and H. F. Roloff, J. Phys. C 10, 473 (1978); F. J.

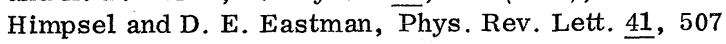
(1978); P. O. Gartland and B. J. Slagsvold, Solid State Commun. 25, 489 (1978); G. V. Hansson and S. A. Flodstrom, Phys. Rev. B 18, 1562 (1978).

${ }^{4}$ F. J. Himpsel, Appl. Opt. $\overline{19}, 3964$ (1980); for symmetry selection rules in the hcp lattice see F. J. Himpsel and D. E. Eastman, Phys. Rev. B 21, 3207 (1980); 22, $5014(1980)$. At $\overrightarrow{\mathrm{k}}_{\|}=0$, the symmetry group is $C_{6 v}$ for bulk states and $C_{3 v}$ for surface states.

${ }^{5}$ D. E. Eastman, J. J. Donelon, N. C. Hien, and F. J. Himpsel, Nuc1. Instrum. Methods 172, 327 (1980).

${ }^{6}$ S. G. Louie, P. Thiry, R. Pinchaux, Y. Petroff,
D. Chandesris, and J. Lecante, Phys. Rev. Lett. 44, 549 (1980).

${ }^{7}$ R. W. Stark and L. M. Falicov, Phys. Rev. Lett. 19, 795 (1967).

${ }^{8}$ P. O. Nilsson, J. Kanski, and C. G. Larsson, Solid State Commun. 36, 111 (1980).

${ }^{9}$ F. J. Himpsel and D. E. Eastman, Phys. Rev. B $\underline{18}$, 5236 (1978).

${ }^{10}$ J. A. Knapp, F. J.Himpse1, and D. E. Eastman, Phys. Rev. B 19, 4952 (1979); P. Thiry, D. Chandesris, J. Lecante, C. Guillot, R. Pinchaux, and Y. Petroff, Phys. Rev. Lett. $\underline{43}, 82$ (1979).

${ }^{11}$ S. G. Louie, Phys. Rev. Lett. 40, 1525 (1978).

${ }^{12}$ P. Heimann, J. Hermanson, H. Miosga, and H. Neddermeyer, Surf. Sci. $\underline{85}, 263$ (1979).

${ }^{13}$ D. E. Eastman, J. A. Knapp, and F. J. Himpsel, Phys. Rev. Lett. 41, 825 (1978); F. J. Himpsel and W. Eberhardt, Solid State Commun. 31, 747 (1979).

${ }^{14} \mathrm{Y}$. Petroff and P. Thiry, Appl. Opt. 19, 3957 (1980). 